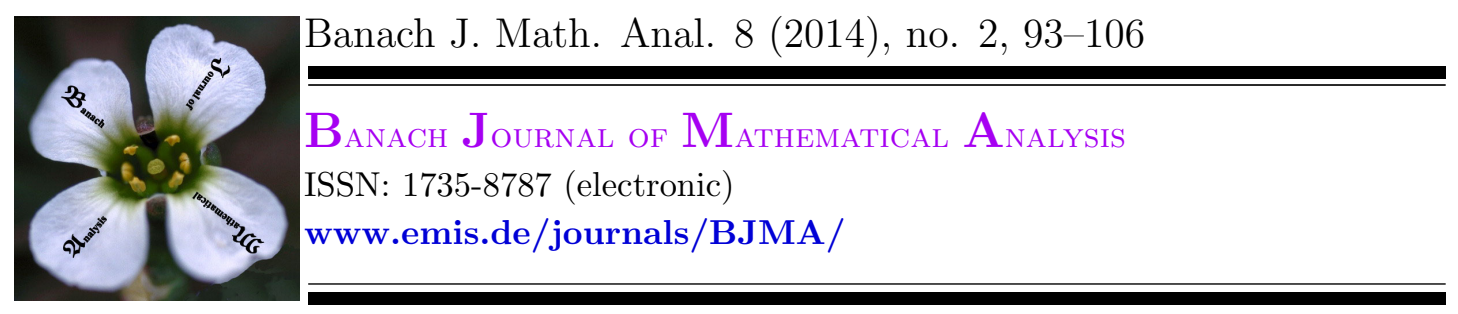

\title{
DISJOINTNESS PRESERVING LINEAR OPERATORS BETWEEN BANACH ALGEBRAS OF VECTOR-VALUED FUNCTIONS
}

\author{
TAHER GHASEMI HONARY*, AZADEH NIKOU AND AMIR HOSSEIN SANATPOUR \\ Communicated by K. Jarosz
}

\begin{abstract}
We present vector-valued versions of two theorems due to A. Jimenez-Vargas, by showing that, if $B(X, E)$ and $B(Y, F)$ are certain vectorvalued Banach algebras of continuous functions and $T: B(X, E) \rightarrow B(Y, F)$ is a separating linear operator, then $\widehat{T}: \widehat{B(X, E)} \rightarrow \widehat{B(Y, F)}$, defined by $\widehat{T} \hat{f}=\widehat{T f}$, is a weighted composition operator, where $\widehat{T f}$ is the Gelfand transform of $T f$.

Furthermore, it is shown that, under some conditions, every bijective separating map $T: B(X, E) \rightarrow B(Y, F)$ is biseparating and induces a homeomorphism between the character spaces $M(B(X, E))$ and $M(B(Y, F))$. In particular, a complete description of all biseparating, or disjointness preserving linear operators between certain vector-valued Lipschitz algebras is provided. In fact, under certain conditions, if the bijections $T: \operatorname{Lip}^{\alpha}(X, E) \rightarrow \operatorname{Lip}^{\alpha}(Y, F)$ and $T^{-1}$ are both disjointness preserving, then $T$ is a weighted composition operator in the form $T f(y)=h(y)(f(\phi(y)))$, where $\phi$ is a homeomorphism from $Y$ onto $X$ and $h$ is a map from $Y$ into the set of all linear bijections from $E$ onto $F$. Moreover, if $T$ is multiplicative then $M(E)$ and $M(F)$ are homeomorphic.
\end{abstract}

\section{INTRODUCTION AND PRELIMINARIES}

Let $X$ be a compact Hausdorff space, $(E,\|\cdot\|)$ be a Banach algebra over the scalar field of complex numbers $\mathbb{C}$ and $C(X, E)$ be the space of all continuous

Date: Received: Jun. 5, 2012; Accepted: Sep. 28, 2013.

* Corresponding author.

2010 Mathematics Subject Classification. Primary 47B38; Secondary 47B33, 47B48, 46J10.

Key words and phrases. Vector-valued Banach algebra, vector-valued Lipschitz algebra, maximal ideal space, disjointness preserving, separating, biseparating. 
maps from $X$ into $E$. We define the uniform norm on $C(X, E)$ by

$$
\|f\|_{X}=\sup _{x \in X}\|f(x)\|, \quad f \in C(X, E) .
$$

For $f, g \in C(X, E)$ and $\lambda \in \mathbb{C}$, the pointwise operations $\lambda f, f+g$ and $f g$ in $C(X, E)$ are defined as usual. It is easy to see that $\left(C(X, E),\|\cdot\|_{X}\right)$ is a Banach algebra. If $E=\mathbb{C}$ we get the ordinary function algebra $C(X, \mathbb{C})=C(X)$ of all continuous complex-valued functions on $X$.

Definition 1.1. Let $(A,\|\cdot\|)$ be a Banach algebra and the character space $M(A)$ denote the set of all characters (nonzero complex-valued multiplicative linear functionals) on $A$.

(i) The Gelfand transform of $f \in A$ is the complex-valued function $\hat{f}$ defined by $\hat{f}(\varphi)=\varphi(f)$ on $M(A)$. Moreover, $\hat{A}=\{\hat{f}: f \in A\}$.

(ii) $A$ is regular if $M(A) \neq \emptyset$ and for every closed subset $F \subseteq M(A)$ and every $\varphi \in M(A) \backslash F$, there exists $f \in A$ such that $\hat{f}(\varphi)=1$ and $\hat{f}(F) \subseteq\{0\}$. If in addition, this $f$ satisfies $\|\hat{f}\| \leq 1$, then $A$ is called hyper-regular.

(iii) $A$ is normal if $M(A) \neq \emptyset$ and for every closed subset $F \subseteq M(A)$ and every compact subset $K \subseteq M(A)$ with $F \cap K=\emptyset$, there exists $f \in A$ such that $\hat{f}(K) \subseteq\{1\}$ and $\hat{f}(F) \subseteq\{0\}$. If in addition, this $f$ satisfies $\|\hat{f}\| \leq 1$, then $A$ is called hyper-normal.

Remark 1.2. (i) A commutative Banach algebra is regular if and only if it is normal. See, for example, [18, Corollary 4.2.9] or [9, Proposition 4.1.18].

(ii) If $A$ is a regular commutative Banach algebra such that $\hat{A}$ is closed under complex conjugation, then $A$ is hyper-regular [18, Corollary 4.2.10].

(iii) Every commutative $C^{*}-$ algebra is regular and hence normal. See, for example, [18, Example 4.2.2]. Moreover, by $(i i)$ every commutative $C^{*}$-algebra is hyper-regular.

Let $X$ be a compact Hausdorff space and $E$ be a unital commutative Banach algebra. In the sequel, by $B(X, E)$ we mean a Banach algebra which is contained in $C(X, E)$. It is clear that if $B(X, E)$ contains the constant functions, then it is commutative if and only if $E$ is commutative. We also recall that the cozero set of $f: X \rightarrow E$ is $\operatorname{coz}(f)=\{x \in X: f(x) \neq 0\}$, and $\operatorname{supp}(f)$, the support of $f$, is the closure of $\operatorname{coz}(f)$ in $X$.

Definition 1.3. For compact Hausdorff spaces $X$ and $Y$, and Banach algebras $\left(E,\|\cdot\|_{E}\right),\left(F,\|\cdot\|_{F}\right)$, a linear map $T: B(X, E) \rightarrow B(Y, F)$ is called disjointness preserving if for every $f, g \in B(X, E)$ the equality $\operatorname{coz}(f) \cap \operatorname{coz}(g)=\emptyset$ implies the equality $\operatorname{coz}(T f) \cap \operatorname{coz}(T g)=\emptyset$.

Remark 1.4. It is easy to check that a linear map $T: B(X, E) \rightarrow B(Y, F)$ is disjointness preserving if and only if for every $f, g \in B(X, E)$ the equality $\|f(x)\|\|g(x)\|=0$ for all $x \in X$ implies the equality $\|T f(y)\|\|T g(y)\|=0$ for all $y \in Y$. If $T$ has this latter property it is called a separating map by some authors. See, for example, [13], [17] and [10]. But in this paper, we use separating maps in the following sense. See, for example, [11] and [12]. 
Definition 1.5. If $A$ and $B$ are Banach algebras, a linear map $T: A \rightarrow B$ is called separating if for every $f, g \in A$, the equality $f g=0$ implies the equality $T f T g=0$. Moreover, $T$ is called biseparating if it is bijective and both $T$ and $T^{-1}$ are separating.

Definition 1.6. Let $A$ and $B$ be Banach algebras and let $T: A \rightarrow B$ be a linear map. The map $\widehat{T}: \hat{A} \rightarrow \hat{B}$ is defined by $\widehat{T} \hat{f}=\widehat{T f}$ for every $f \in A$.

If $A$ and $B$ are semisimple commutative Banach algebras, it is easy to check that the map $T: A \rightarrow B$ is separating if and only if $\widehat{T}$ is separating and, moreover, $T$ is injective (surjective) if and only if $\widehat{T}$ is injective (surjective).

Order bounded disjointness preserving maps are also known as Lamperti operators [4].

The notion of disjointness preserving or separating operators seems to be used first in the 40's $[22,23]$. Since then many mathematicians have developed this concept. For example, Abramovich made some contributions in the context of Banach lattices and vector lattices in [1,2]. Separating linear maps for scalarvalued continuous functions, as well as the notion of automatic continuity, were studied in [5, 6, 7] and for scalar-valued Lipschitz algebras in [16]. Moreover, these maps have been studied in [13] for the algebra of continuous vector-valued functions, as well as the vector-valued Lipschitz algebras. Jarosz has also interesting results on the automatic continuity of separating linear isomorphisms in [15]. Disjointness preserving operators between certain Banach algebras of continuous functions have been studied in [3, 12]. One can also find interesting results on norm-preserving maps between Banach function algebras in [14]. Recently, as examples of weighted composition operators, disjointness preserving maps between vector-valued Lipschitz function spaces have been studied in [10].

In [16] Jimenez-Vargas has shown that for compact metric spaces $X$ and $Y$, every disjointness preserving operator $T: \ell i p^{\alpha}(X) \rightarrow \ell i p^{\alpha}(Y)$ is essentially a weighted composition operator. He also proved that every bijective disjointness preserving operator $T: \ell i p^{\alpha}(X) \rightarrow \ell i p^{\alpha}(Y)$ is automatically continuous and it is, in fact, biseparating.

One of the aims of this paper is to extend the results of Jimenez-Vargas in [16] to Banach algebras of vector-valued continuous functions which are hypernormal, semisimple, commutative and unital. First we require some definitions and notations.

Let $A$ be a unital commutative Banach algebra. The radical of the algebra $A$ is defined to be the intersection of all maximal ideals of $A$ and it is denoted by $\operatorname{rad}(A)$. The algebra $A$ is semisimple if $\operatorname{rad}(A)=\{0\}$.

By using a method similar to Jimenez-Vargas in [16, Theorem 2.2], we show that if $B(X, E)$ and $B(Y, F)$ are hyper-normal, semisimple, commutative and unital, and $T: B(X, E) \rightarrow B(Y, F)$ is a disjointness preserving linear map, then $\widehat{T}$ is a weighted composition operator. Furthermore, with the same conditions, we show that every bijective separating map $T: B(X, E) \rightarrow B(Y, F)$ is biseparating and induces a homeomorphism between the character spaces $M(B(X, E))$ and $M(B(Y, F))$. Then by applying the same method as in [13, Theorem 2.3], we conclude that certain disjointness preserving linear maps $T: \operatorname{Lip}^{\alpha}(X, E) \rightarrow$ 
$\operatorname{Lip}^{\alpha}(Y, F)$ or $T: \ell i p^{\alpha}(X, E) \rightarrow \ell i p^{\alpha}(Y, F)$ are weighted composition operators, and moreover, they induce a homeomorphism between $X$ and $Y$.

Weighted composition operators between certain classes of weighted Frechet spaces and on some spaces of analytic functions, have been studied in [19].

\section{Hyper-normality of VeCtor-VAlued Lipschitz Algebras}

In this section we show that, for a compact metric space $X$ and a commutative unital Banach algebra $E, \operatorname{Lip}^{\alpha}(X, E)\left(\ell i p^{\alpha}(X, E)\right)$ is hyper-normal, or (hyper) regular if and only if $E$ is hyper-normal, or (hyper) regular, respectively. We also show that $E$-valued Lipschitz algebras are semisimple if and only if $E$ is semisimple.

Definition 2.1. Let $(X, d)$ be a compact metric space and $E$ be a unital commutative Banach algebra. For a constant $\alpha(0<\alpha \leq 1)$ and a function $f: X \rightarrow E$, the Lipschitz constant of $f$ is defined by

$$
p_{\alpha}(f):=\sup _{\substack{x, y \in X \\ x \neq y}} \frac{\|f(x)-f(y)\|}{d(x, y)^{\alpha}},
$$

and the vector-valued big Lipschitz algebra (of order $\alpha$ ), or simply, the vectorvalued Lipschitz algebra is defined by

$$
\operatorname{Lip}^{\alpha}(X, E)=\left\{f: X \rightarrow E: p_{\alpha}(f)<\infty\right\} .
$$

Similarly, for $\alpha(0<\alpha<1)$ the vector-valued little Lipschitz algebra (of order $\alpha)$ is defined by

$$
\operatorname{lip}^{\alpha}(X, E)=\left\{f \in \operatorname{Lip}^{\alpha}(X, E): \frac{\|f(x)-f(y)\|}{d(x, y)^{\alpha}} \rightarrow 0 \quad \text { as } \quad d(x, y) \rightarrow 0\right\} .
$$

For each $f \in \operatorname{Lip}^{\alpha}(X, E)$ we define the norm by

$$
\|f\|_{\alpha}=\|f\|_{X}+p_{\alpha}(f) \text {. }
$$

If $E=\mathbb{C}$ we get the ordinary complex-valued Lipschitz algebras $\operatorname{Lip}^{\alpha}(X)$ and $\operatorname{lip}^{\alpha}(X)$. In [8] it has been shown that $\left(\operatorname{Lip}^{\alpha}(X, E),\|\cdot\|_{\alpha}\right)$ is complete and it is, in fact, a Banach subalgebra of $C(X, E)$, and moreover, $\ell i p^{\alpha}(X, E)$ is a closed subalgebra of $\left(\operatorname{Lip}^{\alpha}(X, E),\|\cdot\|_{\alpha}\right)$.

Remark 2.2. For a compact metric space $X$ and a unital commutative Banach algebra $E$, we can deduce from [20, Examples 2.1(ii)] and [20, Corollary 2.2], that the maximal ideal space of $\operatorname{Lip}^{\alpha}(X, E)$ is homeomorphic to the cartesian product $X \times M(E)$ in the product topology, that is,

$$
M\left(\operatorname{Lip}^{\alpha}(X, E)\right) \cong X \times M(E) .
$$

Moreover, every character $\phi$ on $\operatorname{Lip}^{\alpha}(X, E)$ is of the form $\varphi \circ \delta_{x}$ for some $\varphi \in M(E)$ and for some $x \in X[20]$.

We now bring an elementary result for scalar-valued Lipschitz algebras and then extend it to the vector-valued case.

Lemma 2.3. If $X$ is a compact metric space then $\operatorname{Lip}^{\alpha}(X)$ for $0<\alpha \leq 1$ and lip $^{\alpha}(X)$ for $0<\alpha<1$ are both hyper-normal. 
Proof. Since $\operatorname{Lip}^{1}(X)$ is contained in $\ell i p^{\alpha}(X)$ for all $0<\alpha<1$, it is enough to show that for any pair of disjoint compact sets $C$ and $K$ the function

$$
f(x)=\frac{d(x, C)}{d(x, C)+d(x, K)}
$$

is an element of $\operatorname{Lip}^{1}(X)$, which is easy to see.

Theorem 2.4. Let $X$ be a compact metric space and $E$ be a commutative unital Banach algebra. Then $\operatorname{Lip}^{\alpha}(X, E)$ is hyper-normal if and only if $E$ is hypernormal.

Proof. We first suppose that $E$ is hyper-normal. Let $K$ and $F$ be compact subsets of $M\left(\operatorname{Lip}^{\alpha}(X, E)\right)$ such that $K \cap F=\emptyset$. For every $\phi \in K$, there exists a neighbourhood $U_{\phi}$ such that $U_{\phi} \cap F=\emptyset$. By Remark 2.2, there exist $x \in X$ and $\psi \in M(E)$ such that $\phi=\psi \circ \delta_{x}$. Hence there exist neighbourhoods $U_{x}$ and $U_{\psi}$ of $x$ and $\psi$, respectively, such that $\phi \in U_{x} \times U_{\psi} \subseteq U_{\phi}$. Since $X$ and $M(E)$ are compact and Hausdorff, there exist neighbourhoods $V_{x}$ of $x$ and $V_{\psi}$ of $\psi$ such that $x \in V_{x} \subseteq \overline{V_{x}} \subseteq U_{x}$ and $\psi \in V_{\psi} \subseteq \overline{V_{\psi}} \subseteq U_{\psi}$. By Lemma 2.3, $\operatorname{lip}^{\alpha}(X)$ is hyper-normal and hence there exists $f \in \operatorname{lip}^{\alpha}(X)$ such that $0 \leq f(t) \leq 1$ for all $t \in X,\left.f\right|_{\overline{V_{x}}}=0$ and $\left.f\right|_{U_{x}{ }^{c}}=1$. Since $E$ is hyper-normal, there exists $b \in E$ such that $\|\hat{b}\| \leq 1,\left.\hat{b}\right|_{\overline{V_{\psi}}}=0$ and $\left.\hat{b}\right|_{U_{\psi}{ }^{c}}=1$. If we take $g:=b+f e-f b$, where $e$ is the unit element of $E$, then clearly $g \in \operatorname{Lip}^{\alpha}(X, E)$ and $\left.\hat{g}\right|_{V_{x} \times V_{\psi}}=0$. To show that $\|\hat{g}\| \leq 1$ and $\left.\hat{g}\right|_{F}=1$ let $\varphi \in M\left(\operatorname{Lip}^{\alpha}(X, E)\right)$. By Remark 2.2 there exist $\gamma \in M(E)$ and $t \in X$ such that $\varphi=\gamma \circ \delta_{t}$. Thus we have $|\hat{g}(\varphi)|=|\varphi(g)|=|\gamma(g(t))|=|\gamma(b)+f(t)-f(t) \gamma(b)|=|1+(1-f(t))(\gamma(b)-1)|$. If we take $\zeta:=1-f(t)$ and $\beta=\gamma(b)-1$, then $0 \leq \zeta \leq 1$ and $|1+\beta| \leq 1$ and hence $|1+\zeta \beta| \leq 1$. This implies that

$$
|\hat{g}(\varphi)|=|\gamma(b)+f(t)-f(t) \gamma(b)|=|1+(1-f(t))(\gamma(b)-1)|=|1+\zeta \beta| \leq 1 .
$$

Now let $\varphi \in F$. Since $U_{\phi} \cap F=\emptyset$ there exist only five cases as follows:

Case 1: $t \in U_{x}^{c}$ and $\gamma \in U_{\psi}^{c}$. Then $f(t)=1$ and $\gamma(b)=1$ and hence $\hat{g}(\varphi)=1$.

Case 2: $t \in U_{x}{ }^{c}$ and $\gamma \in V_{\psi}$. Then $f(t)=1$ and $\gamma(b)=0$ and hence $\hat{g}(\varphi)=1$.

Case 3: $t \in U_{x}{ }^{c}$ and $\gamma \in U_{\psi} \backslash V_{\psi}$. Then $f(t)=1$ and hence

$$
\hat{g}(\varphi)=\gamma(b)+1-(\gamma(b) \cdot 1)=1 \text {. }
$$

Case 4: $t \in V_{x}$ and $\gamma \in U_{\psi}{ }^{c}$. Then $f(t)=0$ and $\gamma(b)=1$ and hence $\hat{g}(\varphi)=1$. Case 5: $t \in U_{x} \backslash V_{x}$ and $\gamma \in U_{\psi}{ }^{c}$. Then $\gamma(b)=1$ and hence

$$
\hat{g}(\varphi)=1+f(t)-(1 \cdot f(t))=1 .
$$

If $W_{\phi}:=V_{x} \times V_{\psi}$, then for every $\phi \in K$, there exist a neighbourhood $W_{\phi}$ in $M\left(\operatorname{Lip}^{\alpha}(X, E)\right)$ and a function $g \in \operatorname{Lip}^{\alpha}(X, E)$ such that $\left.\hat{g}\right|_{W_{\phi}}=0$ and $\left.\hat{g}\right|_{F}=1$. Since $K$ is compact, there exist $g_{1}, \cdots, g_{n} \in \operatorname{Lip}^{\alpha}(X, E)$ such that $K \subseteq \cup_{i=1}^{n} W_{\phi_{i}}$, $\left.\hat{g}_{i}\right|_{W_{\phi_{i}}}=0$ and $\left.\hat{g}_{i}\right|_{F}=1$ for $i=1, \cdots, n$. If we take $h=g_{1} \cdots g_{n}$, then $h \in$ $\operatorname{Lip}^{\alpha}(X, E),\|\hat{h}\| \leq 1,\left.\hat{h}\right|_{K}=0$ and $\left.\hat{h}\right|_{F}=1$. From this we now conclude that $\operatorname{Lip}^{\alpha}(X, E)$ is hyper-normal.

Conversely, let $\operatorname{Lip}^{\alpha}(X, E)$ be hyper-normal. Let $K$ and $F$ be compact subsets of $M(E)$ such that $K \cap F=\emptyset$. For a fixed element $x$ in $X$, we define $K^{\prime}:=$ 
$\left\{\psi \circ \delta_{x}: \psi \in K\right\}$ and $F^{\prime}:=\left\{\phi \circ \delta_{x}: \phi \in F\right\}$. It is clear that $K^{\prime}$ and $F^{\prime}$ are compact subsets of $M\left(\operatorname{Lip}^{\alpha}(X, E)\right)$ and $K^{\prime} \cap F^{\prime}=\emptyset$. Since $\operatorname{Lip}^{\alpha}(X, E)$ is hypernormal, there exists $f \in \operatorname{Lip}^{\alpha}(X, E)$ such that $\|\hat{f}\| \leq 1,\left.\hat{f}\right|_{K^{\prime}}=1$ and $\left.\hat{f}\right|_{F^{\prime}}=0$. If $b:=f(x)$, then $b \in E$, implying that $\hat{b}(\psi)=\psi(f(x))=\hat{f}\left(\psi \circ \delta_{x}\right)=1$ for every $\psi \in K$. Similarly,

$$
\hat{b}(\phi)=\phi(f(x))=\hat{f}\left(\phi \circ \delta_{x}\right)=0,
$$

for every $\phi \in F$. Since $\|\hat{f}\| \leq 1$, we conclude that $\|\hat{b}\| \leq 1$. Therefore, $E$ is hyper-normal.

By modifying the proof of the theorem above, we also obtain the following result:

Theorem 2.5. Let $X$ be a compact metric space and $E$ be a commutative unital Banach algebra. Then $\operatorname{Lip}^{\alpha}(X, E)$ is (hyper) regular if and only if $E$ is (hyper) regular.

Theorem 2.6. Let $X$ be a compact Hausdorff space, $E$ be a commutative unital Banach algebra and $B(X, E)$ contain the constant functions. Let us suppose that every character on $B(X, E)$ be of the form $\psi \circ \delta_{x}$ for some $\psi \in M(E)$ and $x \in X$, where $\delta_{x}$ is the evaluation homomorphism on $B(X, E)$. Then $B(X, E)$ is semisimple if and only if $E$ is semisimple.

Proof. Since every character $\varphi$ on $B(X, E)$ is of the form $\psi \circ \delta_{x}$ for some $\psi \in M(E)$ and $x \in X$, we have

$$
\operatorname{rad}(B(X, E))=\{f \in B(X, E): \psi(f(x))=0, \psi \in M(E), x \in X\} .
$$

Let $E$ be semisimple and $f \in \operatorname{rad}(B(X, E))$. Then for every character $\varphi$ on $B(X, E)$, we have $\varphi(f)=0$. It follows that $\left(\psi \circ \delta_{x}\right)(f)=\psi(f(x))=0$ for all $x \in X$ and all $\psi \in M(E)$ and hence $f=0$. This implies that $B(X, E)$ is semisimple.

Conversely, let $B(X, E)$ be semisimple and $b \in \operatorname{rad}(E)$. Let $f$ be the constant element of $B(X, E)$, defined by $f(x)=b$ for all $x \in X$. Then for every character $\varphi$ on $B(X, E)$, we have

$$
\varphi(f)=\left(\psi \circ \delta_{x}\right)(f)=\psi(f(x))=\psi(b)=0,
$$

for some $\psi \in M(E)$ and for some $x \in X$. Therefore, $f \in \operatorname{rad}(B(X, E))$ and hence $f=0$. This implies that $E$ is semisimple.

Remark 2.7. Since every character $\varphi$ on $\operatorname{Lip}^{\alpha}(X, E)\left(\ell i p^{\alpha}(X, E)\right)$ is of the form $\psi \circ \delta_{x}$ for some $\psi \in M(E)$ and for some $x \in X$ (see Remark 2.2), by the theorem above the algebra $\operatorname{Lip}^{\alpha}(X, E)\left(\operatorname{lip}^{\alpha}(X, E)\right)$ is semisimple if and only if $E$ is semisimple. These results are also valid for the Banach algebra $C(X, E)$. Moreover, it was shown by Sherbert in [21, Proposition 2.1] that the scalar-valued Lipschitz algebras $\operatorname{Lip}^{\alpha}(X)$ and $\ell i p^{\alpha}(X)$ are regular Banach function algebras. Therefore, they are normal and semisimple. See, for example, [9, Theorem 4.4.24]. 


\section{Separating and Disjointness Preserving Linear Operators}

In [16] Jimenez-Vargas proved that every disjointness preserving linear map between scalar-valued little Lipschitz algebras is a weighted composition operator. We now extend the results of Jimenez-Vargas as follows:

Theorem 3.1. Let $X, Y$ be compact Hausdorff spaces, E, F be unital commutative Banach algebras, and $B(X, E), B(Y, F)$ be hyper-normal semisimple commutative unital Banach algebras.

If $T: B(X, E) \rightarrow B(Y, F)$ is a separating linear map, then

( $i$ ) there exists a disjoint union $M(B(Y, F))=Y_{c} \cup Y_{0} \cup Y_{d}$, where $Y_{0}$ is closed and $Y_{d}$ is open in $M(B(Y, F))$.

(ii) there exists a continuous map $h: Y_{c} \cup Y_{d} \rightarrow M(B(X, E))$ such that $h(\psi) \notin$ $\operatorname{supp}(\hat{f})$ implies $\widehat{T} \hat{f}(\psi)=0$ for all $f \in B(X, E)$.

(iii) there exists a nonvanishing function $k: Y_{c} \rightarrow \mathbb{C}$ such that $\widehat{T} \hat{f}(\psi)=$ $k(\psi) \hat{f}(h(\psi))$ for every $f \in B(X, E)$ and for all $\psi \in Y_{c}$.

(iv) $\widehat{T} \hat{f}(\psi)=0$ for every $f \in B(X, E)$ and for all $\psi \in Y_{0}$.

(v) $h\left(Y_{d}\right)$ is a finite set of nonisolated points of $M(B(X, E))$.

(vi) the functional $\delta_{\psi} \circ \widehat{T}$ is discontinuous on $\widehat{B(X, E)}$ for each $\psi \in Y_{d}$.

Proof. We divide the set $M(B(Y, F))$ into three disjoint parts: Its null part

$$
Y_{0}:=\left\{\psi \in M(B(Y, F)): \delta_{\psi} \circ \widehat{T}=0\right\},
$$

its nonnull continuous part

$Y_{c}:=\left\{\psi \in M(B(Y, F)): \delta_{\psi} \circ \widehat{T}: \widehat{B(X, E)} \rightarrow \mathbb{C}\right.$ is continuous and nonzero $\}$, and its discontinuous part

$Y_{d}:=\left\{\psi \in M(B(Y, F)): \delta_{\psi} \circ \widehat{T}: \widehat{B(X, E)} \rightarrow \mathbb{C}\right.$ is discontinuous $\}$.

The proof of the theorem is set out, step by step. For steps 2, 3, 5 and 6 , we follow the same method as in the proof of [16, Theorem 2.2] for $\widehat{T}$, instead of $T$, while presenting a different method for the proof of the other steps. We provide all the details for the sake of completeness.

Step 1. For each $\psi \in Y_{c} \cup Y_{d}, \operatorname{supp}\left(\delta_{\psi} \circ \widehat{T}\right) \neq \emptyset$ and, in fact, it contains exactly one point.

Proof. Since $B(X, E)$ and $B(Y, F)$ are hyper-normal and semisimple commutative unital Banach algebras, by [11, Lemma 1], for every $\psi \in M(B(Y, F))$, there exists $f_{\psi} \in B(X, E)$ with $\widehat{T}\left(\hat{f}_{\psi}\right)(\psi) \neq 0$. Hence $\operatorname{supp}\left(\delta_{\psi} \circ \widehat{T}\right)$ contains exactly one point for every $\psi \in Y_{c} \cup Y_{d}$.

The map $h: Y_{c} \cup Y_{d} \rightarrow M(B(X, E))$, defined by $h(\psi)=\operatorname{supp}\left(\delta_{\psi} \circ \widehat{T}\right)$, is called the support map of $\widehat{T}$.

Step 2. If $\psi \in Y_{c} \cup Y_{d}, f \in B(X, E)$ and $h(\psi) \notin \operatorname{supp}(\hat{f})$, then $\widehat{T} \hat{f}(\psi)=0$.

Proof. If $h(\psi) \notin \operatorname{supp}(\hat{f})$, then there exists $U_{h(\psi)}$ such that $\hat{f}(\phi)=0$ if $\phi \in U_{h(\psi)}$. Since $h(\psi)=\operatorname{supp}\left(\delta_{\psi} \circ \widehat{T}\right)$, there is a function $g \in B(X, E)$ such that $\widehat{T} \hat{g}(\psi) \neq 0$ and $\hat{g}(\phi)=0$ if $\phi \notin U_{h(\psi)}$, implying that $\hat{f}(\phi) \hat{g}(\phi)=0$ for all $\phi \in M(B(X, E))$. 
Since $T$ is separating, $\widehat{T}$ is also separating. This implies that $\widehat{T} \hat{f}(\psi) \widehat{T} \hat{g}(\psi)=0$ and hence $\widehat{T} \hat{f}(\psi)=0$.

Step 3. The map $h: Y_{c} \cup Y_{d} \rightarrow M(B(X, E))$ is continuous in the weak*topology.

Proof. Let $\psi$ be in $Y_{c} \cup Y_{d}$ and $\left\{\psi_{\gamma}\right\}_{\gamma \in I}$ be a net in $Y_{c} \cup Y_{d}$ converging to $\psi$. Towards a contradiction, suppose that $\left\{h\left(\psi_{\gamma}\right)\right\}_{\gamma \in I}$ does not converge to $h(\psi)$. Then there exists a neighbourhood $N_{h(\psi)}$ and a subnet $\left\{h\left(\psi_{\lambda}\right)\right\}_{\lambda \in J}$ of $\left\{h\left(\psi_{\gamma}\right)\right\}_{\gamma \in I}$ such that $\left\{h\left(\psi_{\lambda}\right)\right\} \notin N_{h(\psi)}$ for each $\lambda \in J$.

By the compactness of $M(B(X, E))$ there is a subnet $\left\{h\left(\psi_{\beta}\right)\right\}_{\beta \in K}$ of $\left\{h\left(\psi_{\lambda}\right)\right\}_{\lambda \in J}$ which is convergent to an element $\phi \in M(B(X, E))$. If $\phi \neq h(\psi)$, then there exist neighbourhoods $V, W$ of $h(\psi)$ and $\phi$, respectively, such that $V \cap W=\emptyset$. Since $\left\{h\left(\psi_{\beta}\right)\right\}_{\beta \in K}$ converges to $\phi$, there exists $\beta_{0} \in K$ such that $h\left(\psi_{\beta}\right) \in W$ if $\beta \geq \beta_{0}$. Since $h(\psi)=\operatorname{supp}\left(\delta_{\psi} \circ \widehat{T}\right)$, there exists a function $f \in B(X, E)$ such that $\hat{f}(\lambda)=0$ for all $\lambda \notin V$ and $\widehat{T} \hat{f}(\psi) \neq 0$. Thus $\hat{f}(\lambda)=0$ for every $\lambda \in W$. In particular, $h\left(\psi_{\beta}\right) \notin \operatorname{supp}(\hat{f})$ and hence $\widehat{T} \hat{f}\left(\psi_{\beta}\right)=0$ for all $\beta \geq \beta_{0}$, by Step 2. Thus $\widehat{T} \hat{f}(\psi)=0$, which is a contradiction. Consequently, $h\left(\psi_{\beta}\right) \rightarrow_{\beta \in K} h(\psi)$. Since $\{h(\psi)\}_{\beta \in K}$ is a subnet of $\left\{h\left(\psi_{\lambda}\right)\right\}_{\lambda \in J}$, it follows that $h\left(\psi_{\beta}\right) \notin N_{h(\psi)}$ for all $\beta \in K$, which is impossible. Therefore, $\left\{h\left(\psi_{\gamma}\right)\right\}_{\gamma \in I}$ converges to $h(\psi)$, implying that $h$ is continuous.

Step 4. For $\psi \in Y_{c} \cup Y_{d}$, let

$M_{\psi}:=\{\hat{f} \in \widehat{B(X, E)}: \hat{f}(h(\psi))=0\}, \quad J_{\psi}:=\{\hat{f} \in \widehat{B(X, E)}: h(\psi) \notin \operatorname{supp}(\hat{f})\}$.

Then $J_{\psi}$ is a dense subspace of $M_{\psi}$.

Proof. Note that $J_{\psi}$ is, in fact, the set all functions in $\widehat{B(X, E)}$ vanishing on a neighbourhood of $h(\psi)$. Clearly $J_{\psi}$ and $M_{\psi}$ are vector subspaces of $\widehat{B(X, E)}$ and $J_{\psi} \subseteq M_{\psi}$. To show that $J_{\psi}$ is dense in $M_{\psi}$, let $\psi \in Y_{c} \cup Y_{d}, \hat{f} \in M_{\psi}$ and $\epsilon>0$. Define

$\Gamma_{1}:=\left\{\phi \in M(B(X, E)):|\hat{f}(\phi)| \leq \frac{\epsilon}{2}\right\}, \quad \Gamma_{2}:=\{\phi \in M(B(X, E)):|\hat{f}(\phi)| \geq \epsilon\}$.

Since $B(X, E)$ is hyper-normal, there exists $g \in B(X, E)$ such that $\|\hat{g}\| \leq 1$, $\left.\hat{g}\right|_{\Gamma_{1}}=0$ and $\left.\hat{g}\right|_{\Gamma_{2}}=1$. Since the interior of $\Gamma_{1}$ is a neighbourhood of $h(\psi)$ and $\hat{g}$ is zero on this neighbourhood, it follows that $\hat{g} \in J_{\psi}$ and hence $\hat{f} \hat{g} \in J_{\psi}$.

We now consider the following three cases:

Case 1: If $\phi \in \Gamma_{1}$, then $|\hat{f}(\phi)(1-\hat{g}(\phi))| \leq \frac{\epsilon}{2}(1+\|\hat{g}\|)<\epsilon$.

Case 2: If $\phi \in \Gamma_{2}{ }^{c} \backslash \Gamma_{1}$, then $|\hat{f}(\phi)(1-\hat{g}(\phi))| \leq \epsilon(1+\|\hat{g}\|)<2 \epsilon$.

Case 3: If $\phi \in \Gamma_{2}$, then $|\hat{f}(\phi)(1-\hat{g}(\phi))|=0$.

Therefore, $\|\hat{f}-\hat{f} \hat{g}\|<2 \epsilon$, implying that $J_{\psi}$ is dense in $M_{\psi}$.

Step 5. There exists a nonvanishing function $k: Y_{c} \rightarrow \mathbb{C}$ such that

$$
\widehat{T} \hat{f}(\psi)=k(\psi) \hat{f}(h(\psi)),
$$

for all $f \in B(X, E)$ and all $\psi \in Y_{c}$. 
Proof. Let $\psi \in Y_{c}$. Since $\delta_{\psi} \circ \widehat{T}$ is a nonzero continuous linear functional on $\widehat{B(X, E)}$, it follows that $\operatorname{ker}\left(\delta_{\psi} \circ \widehat{T}\right)$ is a proper closed subspace of $\widehat{B(X, E)}$ and moreover, $J_{\psi} \subset \operatorname{ker}\left(\delta_{\psi} \circ \widehat{T}\right)$ by Step 2. Therefore, $\operatorname{ker} \delta_{h(\psi)}=M_{\psi} \subset \operatorname{ker}\left(\delta_{\psi} \circ \widehat{T}\right)$ by Step 4 . Hence there exists a nonzero scalar $k(\psi)$ such that $\delta_{\psi} \circ \widehat{T}=k(\psi) \delta_{h(\psi)}$, implying that $\widehat{T} \hat{f}(\psi)=k(\psi) \hat{f}(h(\psi))$ for all $f \in B(X, E)$.

Step 6. The set $Y_{0}$ is closed in $M(B(Y, F))$ and the set $Y_{d}$ is open in $M(B(Y, F))$. Proof. Since $Y_{0}=\cap_{f \in B(X, E)} \operatorname{ker}(\widehat{T} \hat{f})$, it follows that $Y_{0}$ is closed in $M(B(Y, F))$. To show that $Y_{d}$ is open in $M(B(Y, F))$, let $\left\{\psi_{\gamma}\right\}_{\gamma \in I}$ be a net in $M(B(Y, F)) \backslash Y_{d}$, which converges to a point $\psi \in M(B(Y, F))$. By Step 5 , there exists a nonvanishing bounded function $k: Y_{c} \rightarrow \mathbb{C}$ such that

$$
\begin{aligned}
\left|\widehat{T} \hat{f}\left(\psi_{\gamma}\right)\right| & \leq \sup \left\{|\widehat{T} \hat{f}(\psi)|: \psi \in Y_{0} \cup Y_{c}\right\} \leq \sup \left\{|\widehat{T} \hat{f}(\psi)|: \psi \in Y_{c}\right\} \\
& \leq \sup \left\{|k(\psi) \hat{f}(h(\psi))|: \psi \in Y_{c}\right\} \leq\|k\|\|\hat{f}\|,
\end{aligned}
$$

for all $f \in B(X, E)$ and $\gamma \in I$, where $\|k\|$ is the supremum norm of $k$. However, for the boundedness of $k$ we may take $f=1_{E}$, the unit element of $B(X, E)$, in $\widehat{T} \hat{f}(\psi)=k(\psi) \hat{f}(h(\psi))$ and conclude that $k$ is bounded. By the continuity of $\widehat{T} \hat{f}$ on $M(B(Y, F))$, we have $|\widehat{T} \hat{f}(\psi)| \leq\|k\|\|\hat{f}\|$, that is, $\left|\delta_{\psi} \circ \widehat{T}(\hat{f})\right| \leq\|k\|\|\hat{f}\|$. Thus the linear functional $\delta_{\psi} \circ \widehat{T}$ is continuous on $\widehat{B(X, E)}$ and hence $\psi \in M(B(Y, F)) \backslash Y_{d}$. This shows that $M(B(Y, F)) \backslash Y_{d}$ is closed and hence $Y_{d}$ is open in $M(B(Y, F))$.

Step 7. $h\left(Y_{d}\right)$ is a finite set of nonisolated points of $M(B(X, E))$.

Proof. For the finiteness of $h\left(Y_{d}\right)$, let $\left(h\left(\psi_{n}\right)\right)_{n \in \mathbb{N}}$ be a sequence of distinct elements of $M(B(X, E))$ such that $\psi_{n} \in Y_{d}$ for all $n \in \mathbb{N}$. Moreover, suppose that there exist sequences $\left(V_{n}\right)_{n \in \mathbb{N}}$ and $\left(U_{n}\right)_{n \in \mathbb{N}}$ of pairwise disjoint neighbourhoods of $h\left(\psi_{n}\right)$ such that $U_{n} \subseteq \overline{U_{n}} \subseteq V_{n}$ for all $n \in \mathbb{N}$. Since $B(X, E)$ is hyper-normal, for each $n$, there exists $g_{n} \in B(X, E)$ such that $\hat{g}_{n}=1$ on $U_{n}$ and $\operatorname{supp}\left(\hat{g}_{n}\right) \subseteq V_{n}$. On the other hand, since the linear functional $\delta_{\psi_{n}} \circ \widehat{T}$ is discontinuous on $\widehat{B(X, E)}$, there exists a function $h_{n} \in B(X, E)$ with $\left\|h_{n}\right\| \leq 1$ such that $\left|\widehat{T} \hat{h}_{n}\left(\psi_{n}\right)\right| \geq n^{3}\left\|g_{n}\right\|$ for all $n \in \mathbb{N}$. If $f_{n}:=\frac{g_{n} h_{n}}{n^{2}\left\|g_{n}\right\|}$ for $n \in \mathbb{N}$, then $\hat{f}_{n}-\frac{\hat{h}_{n}}{n^{2}\left\|g_{n}\right\|}=0$ on $U_{n}$, implying that $h\left(\psi_{n}\right) \notin \operatorname{supp}\left(\hat{f}_{n}-\frac{\hat{h}_{n}}{n^{2}\left\|g_{n}\right\|}\right)$. Hence $\left|\widehat{T} \hat{f}_{n}\left(\psi_{n}\right)\right|=\frac{1}{n^{2}\left\|g_{n}\right\|}\left|\widehat{T} \hat{h}_{n}\left(\psi_{n}\right)\right|$ by Step 2, so that $\left|\widehat{T} \hat{f}_{n}\left(\psi_{n}\right)\right| \geq n$. Since $B(X, E)$ is complete and $\left\|f_{n}\right\|<\frac{1}{n^{2}}$ for all $n \in \mathbb{N}$, we can define the function $f=\sum_{n=1}^{\infty} f_{n} \in B(X, E)$. From the fact that the Gelfand transform is a linear continuous mapping, we deduce $\hat{f}=\sum_{n=1}^{\infty} \hat{f}_{n}$. Since the sequence $\left(V_{n}\right)_{n \in \mathbb{N}}$ is pairwise disjoint and $\operatorname{coz}\left(\hat{f}_{n}\right) \subseteq V_{n}$ for all $n \in \mathbb{N}$, it follows that $h\left(\psi_{m}\right) \notin \operatorname{supp}\left(\hat{f}_{n}\right)$ for all $n \neq m$.

We now show that $h\left(\psi_{m}\right) \notin \operatorname{supp}\left(\sum_{n=1, n \neq m}^{\infty} \hat{f}_{n}\right)$. If $h\left(\psi_{m}\right) \in \operatorname{supp}\left(\sum_{n=1, n \neq m}^{\infty} \hat{f}_{n}\right)$ then

$$
h\left(\psi_{m}\right) \in \overline{\operatorname{coz}\left(\sum_{n=1, n \neq m}^{\infty} \hat{f}_{n}\right)} \subseteq \overline{\cup_{n=1, n \neq m}^{\infty} \operatorname{coz}\left(\hat{f}_{n}\right)} .
$$


Since $V_{m}$ is an open neighbourhood of $h\left(\psi_{m}\right)$, there exists an element $\varphi_{m} \in$ $\cup_{n=1, n \neq m}^{\infty} \operatorname{coz}\left(\hat{f}_{n}\right)$ such that $\varphi_{m} \in V_{m}$. On the other hand, there exists $n \neq m$ such that $\varphi_{m} \in \operatorname{coz}\left(\hat{f}_{n}\right) \subseteq V_{n}$, which is a contradiction, since $V_{n} \cap V_{m}=\emptyset$.

By Step 2 we conclude that $\delta_{\psi_{m}} \circ \hat{T}\left(\sum_{n=1, n \neq m}^{\infty} \hat{f}_{n}\right)=0$. Since

$$
\hat{f}=\hat{f}_{m}+\sum_{n=1, n \neq m}^{\infty} \hat{f}_{n}
$$

it follows that $\delta_{\psi_{m}} \circ \hat{T}(\hat{f})=\delta_{\psi_{m}} \circ \hat{T}\left(\hat{f}_{m}\right)$. Therefore,

$$
\left|\widehat{T} \hat{f}\left(\psi_{m}\right)\right|=\left|\widehat{T} \hat{f}_{m}\left(\psi_{m}\right)\right| \geq m,
$$

for all $m \in \mathbb{N}$, which is a contradiction, since $\widehat{T} \hat{f} \in \widehat{B(Y, F)}$ is bounded. This proves that $h\left(Y_{d}\right)$ is finite.

We now show that each point of $h\left(Y_{d}\right)$ is a nonisolated point of $M(B(X, E))$. Let $h(\psi)$ be an isolated point of $M(B(X, E))$ for some $\psi \in Y_{d}$. Then there exists a neighbourhood $U_{h(\psi)}$ such that $U_{h(\psi)}=\{h(\psi)\}$. If $\hat{f}(h(\psi))=0$, then $h(\psi) \notin$ $\operatorname{supp}(\hat{f})$ and hence $\widehat{T} \hat{f}(\psi)=0$, by Step 2. In other words, $\operatorname{ker}\left(\delta_{h(\psi)}\right) \subseteq \operatorname{ker}\left(\delta_{\psi} \circ \widehat{T}\right)$ and therefore, $\delta_{\psi} \circ \widehat{T}=\beta_{\psi} \delta_{h(\psi)}$ for some nonzero scalar $\beta_{\psi}$. Consequently, the nonzero linear functional $\delta_{\psi} \circ \widehat{T}$ is continuous on $\widehat{B(X, E)}$ and hence $\psi \in Y_{c}$, which is a contradiction.

The proof of the theorem is now complete.

Note that the method of Jimenez-Vargas in [16] is only valid for the Lipschitz algebras, whereas by our method, the same results are valid for more general classes of vector-valued Banach algebras. We are now ready to prove that, under the same conditions as in the theorem above, every separating linear bijection between certain Banach algebras of vector-valued functions is biseparating. This is the most important part of the following theorem:

Theorem 3.2. Let $X, Y$ be compact Hausdorff spaces, $E, F$ be unital commutative Banach algebras and $B(X, E), B(Y, F)$ be hyper-normal semisimple commutative unital Banach algebras. Let $T$ be a separating linear bijection from $B(X, E)$ onto $B(Y, F)$. Then $\widehat{T}$ is a weighted composition operator in the form $\widehat{T} \hat{f}(\psi)=$ $k(\psi) \hat{f}(h(\psi))$ for all $f \in B(X, E)$ and for all $\psi \in M(B(Y, F))$, where $k \in \widehat{B(Y, F})$ is a nonvanishing function and $h$ is a homeomorphism from $M(B(Y, F))$ onto $M(B(X, E))$. In particular, $T$ is biseparating.

Proof. We adopt the same notations as in the previous theorem and divide the proof into several parts.

Part 1. $Y_{0}=\emptyset$ and $Y_{c}$ is compact.

Proof. Let $\psi \in Y_{0}$. Then $\delta_{\psi} \circ \widehat{T}=0$ and $\delta_{\psi}(\widehat{T} \hat{f})=0$ for every $f \in B(X, E)$. Since $T$ is surjective, $\widehat{T}$ is also surjective and hence for every $g \in B$, there exists $f \in B(X, E)$ such that $\hat{g}=\widehat{T} \hat{f}$. Thus $\delta_{\psi}(\hat{g})=\psi(g)=0$ for all $g \in B$ and hence $\psi=0$, which is impossible. Therefore, $Y_{0}=\emptyset$. 
Since by Theorem 3.1, the set $Y_{d}$ is open in $M(B(Y, F))$, it follows that $Y_{c}=$ $M(B(Y, F)) \backslash Y_{d}$ is closed and hence it is compact in $M(B)$.

Part 2. The set $h\left(Y_{c}\right)$ is dense in $M(B(X, E))$.

Proof. We first prove that $h\left(Y_{c} \cup Y_{d}\right)$ is dense in $M(B(X, E))$. Suppose, on the contrary, that there exists a point $\phi \in M(B(X, E))$ such that $V_{\phi} \cap h\left(Y_{c} \cup Y_{d}\right)=\emptyset$, where $V_{\phi}$ is a neighbourhood of $\phi$. Let $U_{\phi}$ be a neighbourhood of $\phi$ such that $U_{\phi} \subseteq \overline{U_{\phi}} \subseteq V_{\phi}$ and $h_{\phi}$ be a nonzero function in $B(X, E)$ such that $\operatorname{supp}\left(\hat{h}_{\phi}\right) \subseteq \overline{U_{\phi}}$. Hence $h(\psi) \notin \operatorname{supp}\left(\hat{h}_{\phi}\right)$ for all $\psi \in Y_{c} \cup Y_{d}$, implying that $\widehat{T} \hat{h}_{\phi}(\psi)=0$ for all $\psi \in Y_{c} \cup Y_{d}$, by Theorem 3.1. Since $Y_{0}$ is empty, $\widehat{T} \hat{h}_{\phi}=0$. By the linearity and injectivity of $\widehat{T}$, it follows that $\hat{h}_{\phi}=0$. Since $B(X, E)$ is semisimple, $h_{\phi}=0$, which is impossible.

We now show that $\overline{h\left(Y_{c} \cup Y_{d}\right)}=\overline{h\left(Y_{c}\right)}$. It suffices to prove that $h\left(Y_{d}\right) \subseteq \overline{h\left(Y_{c}\right)}$. Let $\phi \in h\left(Y_{d}\right)$ and there exist a neighbourhood $U_{\phi}$ of $\phi$ such that $U_{\phi} \cap h\left(Y_{c}\right)=\emptyset$. Since $h\left(Y_{d}\right)$ is finite, there exists a neighbourhood $V_{\phi}$ of $\phi$ such that

$$
V_{\phi} \backslash\{\phi\} \cap h\left(Y_{c} \cup Y_{d}\right)=\emptyset .
$$

Since by Theorem 3.1, $\phi$ is a nonisolated point of $M(B(X, E))$, there is a point $\psi$ in $V_{\phi} \backslash\{\phi\}$. Let $V_{\psi}$ be a neighbourhood of $\psi$ such that $V_{\psi} \subseteq V_{\phi} \backslash\{\phi\}$. Then $V_{\psi} \cap h\left(Y_{c} \cup Y_{d}\right)=\emptyset$ and this contradicts the density of $h\left(Y_{c} \cup Y_{d}\right)$ in $M(B(X, E))$. Hence $\overline{h\left(Y_{c} \cup Y_{d}\right)}=\overline{h\left(Y_{c}\right)}$.

Part 3. $Y_{d}$ is empty.

Proof. Let $\phi \in Y_{d}$. Since $Y_{c}$ is closed, there exists a neighbourhood $V_{\phi}$ such that $V_{\phi} \cap Y_{c}=\emptyset$. By the normality of $B(X, E)$, there exists a function $h_{\phi}$ in $B(Y, F)$ such that $\hat{h}_{\phi}(\phi)=1$ and $\operatorname{coz}\left(\hat{h}_{\phi}\right) \subseteq V_{\phi}$. By the surjectivity of $\widehat{T}$, there exists some $f$ in $B(X, E)$ such that $\widehat{T} \hat{f}=\hat{h}_{\phi}$. Then $\widehat{T} \hat{f}(\psi)=\hat{h}_{\phi}(\psi)=0$ for all $\psi \in Y_{c}$. By Theorem 3.1, $\widehat{T} \hat{f}(\psi)=k(\psi) \hat{f}(h(\psi))$ for all $\psi \in Y_{c}$. Since $T$ is surjective, $k(\psi) \neq 0$ for all $\psi \in Y_{c}$. Hence $\hat{f}(\lambda)=0$ for all $\lambda \in M(B(X, E))$, since $\overline{h\left(Y_{c}\right)}=M(B(X, E))$ by Part 2. Therefore, $\hat{f}=0$ and $\widehat{T} \hat{f}=0$, but $\widehat{T} \hat{f}(\phi)=\hat{h}_{\phi}(\phi)=1$, which is a contradiction.

Part 4. $M(B(Y, F))=Y_{c}$ and $\widehat{T} \hat{f}(\psi)=k(\psi) \hat{f}(h(\psi))$ for all $f \in B(X, E)$ and $\psi \in M(B(Y, F))$.

Proof. By Parts 1 and 3, $Y_{0}=Y_{d}=\emptyset$. Hence $M(B(Y, F))=Y_{c}$ and the result follows from Theorem 3.1.

Part 5. $T^{-1}$ is separating and hence $T$ is biseparating.

Proof. Let $g_{1}, g_{2} \in B(Y, F)$ such that $\hat{g}_{1} \hat{g}_{2}=0$. Then there exist $f_{1}, f_{2} \in B(X, E)$ such that $\hat{g}_{1}=\hat{T} \hat{f}_{1}=k\left(\hat{f}_{1} \circ h\right), \hat{g}_{2}=\hat{T} \hat{f}_{2}=k\left(\hat{f}_{2} \circ h\right)$ and hence $k^{2}\left(\hat{f}_{1} \circ h\right)\left(\hat{f}_{2} \circ h\right)=0$. Since $k(\psi) \neq 0$ for every $\psi \in M(B(Y, F))$, we have $\left(\hat{f}_{1} \hat{f}_{2}\right) \circ h=0$. By Parts 2,4 and the density of $h(M(B(Y, F)))$ in $M(B(X, E))$, it follows that $\hat{f}_{1} \hat{f}_{2}=0$ and hence $\widehat{T^{-1}}$ is separating. Since $B(X, E)$ and $B(Y, F)$ are semisimple commutative 
Banach algebras, $T^{-1}: B(Y, F) \rightarrow B(X, E)$ is separating if and only if $\widehat{T^{-1}}$ : $\widehat{B(Y, F)} \rightarrow \widehat{B(X, E)}$ is separating. Consequently, $T^{-1}$ is separating.

Part 6. The map $h$ is a homeomorphism from $M(B(Y, F))$ onto $M(B(X, E))$.

Proof. For the injectivity of $h: M(B(Y, F)) \rightarrow M(B(X, E))$, let $\phi, \psi$ be elements of $M(B(Y, F))$ with $\phi \neq \psi$ and $h(\phi)=h(\psi)$. Let $U_{\psi}$ be a neighbourhood of $\psi$ such that $\phi \notin U_{\psi}$. Consider $h_{\psi} \in B(Y, F)$ such that $\hat{h}_{\psi}(\psi)=1$ and $\operatorname{coz}\left(\hat{h}_{\psi}\right) \subseteq U_{\psi}$. Since $h_{\psi} \in B(Y, F)$ and $\widehat{T}$ is surjective, $\widehat{T} \hat{f}=\hat{h}_{\psi}$ for some $f \in B(X, E)$. By Part 4 we have

$$
\hat{h}_{\psi}(\lambda)=\widehat{T} \hat{f}(\lambda)=k(\lambda) \hat{f}(h(\lambda)) \quad(\lambda \in M(B(Y, F))) .
$$

In particular, $1=\hat{h}_{\psi}(\psi)=k(\psi) \hat{f}(h(\psi))$ and $0=\hat{h}_{\psi}(\phi)=k(\phi) \hat{f}(h(\phi))$. Hence $\hat{f}(h(\psi))=1 / k(\psi)$ and $\hat{f}(h(\phi))=0$. Since $h(\psi)=h(\phi)$, we get a contradiction. On the other hand, by Parts 2 and $4, \overline{h(M(B(Y, F)))}=M(B(X, E))$ and hence $h$ is continuous by Theorem 3.1. Since $M(B(Y, F))$ is compact, it follows that $h(M(B(Y, F)))=M(B(X, E))$. Therefore, $h: M(B(Y, F)) \rightarrow M(B(X, E))$ is surjective.

The proof of the theorem is now complete.

We should mention here that the proof of the theorem above follows closely [16, Theorem 3.1], except for the continuity of $T$. One may also compare this theorem with [13, Theorem 2.3].

Remark 3.3. By applying the results of Section 2, we conclude that if $E$ and $F$ are semisimple hyper-normal unital commutative Banach algebras, then the Lipschitz algebras $\operatorname{Lip}^{\alpha}(X, E)$ and $\ell i p^{\alpha}(X, E)$ possess the same properties. Hence in this case big and little Lipschitz algebras are interesting examples satisfying the hypotheses of Theorems 3.1 and 3.2.

In [10] Esmaeili and Mahyar characterized disjointness preserving bounded linear operators between spaces of vector-valued little Lipschitz functions on compact metric spaces. In fact, they have shown that every disjointness preserving bounded linear operator between spaces of vector-valued little Lipschitz functions is a weighted composition operator.

In the following, disjointness preserving linear operators between big and little vector-valued Lipschitz algebras are characterized, without the boundedness condition.

Theorem 3.4. [17, Theorems 3.1 and 4.1] Let $X, Y$ be compact metric spaces and $E, F$ be Banach algebras. Let $T$ be a bijection from $\operatorname{Lip}^{\alpha}(X, E)\left(\ell^{\alpha} p^{\alpha}(X, E)\right)$ onto $\operatorname{Lip}^{\alpha}(Y, F)\left(\ell i p^{\alpha}(Y, F)\right)$ such that both $T$ and $T^{-1}$ are disjointness preserving maps. Then $T$ is a weighted composition operator in the form

$$
T f(y)=h(y)(f(\phi(y))), \quad\left(y \in Y, f \in \operatorname{Lip}^{\alpha}(X, E)\left(\ell i p^{\alpha}(X, E)\right)\right),
$$

where $\phi$ is a homeomorphism from $Y$ onto $X$ and $h(y)$ is an invertible linear map from $E$ onto $F$ for each $y \in Y$. Moreover, $T$ is bounded if and only if $h(y)$ is bounded for all $y \in Y$. 
Corollary 3.5. Let $X, Y$ be compact metric spaces and $E, F$ be Banach algebras. If $T$ is a bijection from $\operatorname{Lip}^{\alpha}(X, E)\left(\ell i p^{\alpha}(X, E)\right)$ onto $\operatorname{Lip}^{\alpha}(Y, F)\left(\ell i p^{\alpha}(Y, F)\right)$, such that both $T$ and $T^{-1}$ are disjointness preserving, then $X$ is homeomorphic to $Y$. In particular, if $T$ is multiplicative then $M(E)$ is homeomorphic to $M(F)$.

Proof. By Theorem 3.4, $X$ is homeomorphic to $Y$. In the case that $T$ is multiplicative we actually show that $M(E)$ is homeomorphic to $M(F)$. For this purpose, let $y_{0}$ be a fixed element of $Y$ and define $\lambda: M(F) \rightarrow M(E)$ by $\lambda(\psi)=\psi \circ h\left(y_{0}\right)$. We first show that $\lambda$ is well-defined.

By Theorem 3.4, $T$ is a weighted composition operator in the form

$$
T f\left(y_{0}\right)=h\left(y_{0}\right)\left(f\left(\phi\left(y_{0}\right)\right)\right), \quad\left(f \in \operatorname{Lip}^{\alpha}(X, E)\left(\ell i p^{\alpha}(X, E)\right)\right),
$$

where $\phi$ is a homeomorphism from $Y$ onto $X$ and $h(y)$ is an invertible linear map from $E$ onto $F$ for each $y \in Y$. First we show that $h\left(y_{0}\right)$ is, in fact, a homomorphism. To this end, let $a, b \in E$ and take the constants functions $f=a, g=b$ in $\operatorname{Lip}^{\alpha}(X, E)$. Since $T$ is multiplicative, we have

$$
\begin{aligned}
h\left(y_{0}\right)(a b) & =h\left(y_{0}\right)\left(f\left(\phi\left(y_{0}\right)\right) g\left(\phi\left(y_{0}\right)\right)\right)=h\left(y_{0}\right)\left(f g\left(\phi\left(y_{0}\right)\right)\right)=\operatorname{Tf} g\left(y_{0}\right) \\
& =T f\left(y_{0}\right) \operatorname{Tg}\left(y_{0}\right)=h\left(y_{0}\right)\left(f\left(\phi\left(y_{0}\right)\right)\right) h\left(y_{0}\right)\left(g\left(\phi\left(y_{0}\right)\right)\right) \\
& =h\left(y_{0}\right)(a) h\left(y_{0}\right)(b) .
\end{aligned}
$$

Therefore, $h\left(y_{0}\right)$ is a homomorphism and since $h\left(y_{0}\right)$ is a linear map, it follows that $\psi \circ h\left(y_{0}\right)$ is a homomorphism for all $\psi \in M(F)$. Since $\psi$ is a character, there exists $b \in F$ such that $\psi(b) \neq 0$ and since $h\left(y_{0}\right)$ is onto, we have $h\left(y_{0}\right)(a)=b$, for some $a \in E$. Therefore, $\psi \circ h\left(y_{0}\right)(a) \neq 0$ and hence $\psi \circ h\left(y_{0}\right) \in M(E)$, which implies that $\lambda$ is well defined.

For the injectivity of $\lambda$, let $\lambda\left(\psi_{1}\right)=\lambda\left(\psi_{2}\right)$. Then $\psi_{1} \circ h\left(y_{0}\right)=\psi_{2} \circ h\left(y_{0}\right)$ and hence for every $a \in E, \psi_{1}\left(h\left(y_{0}\right)(a)\right)=\psi_{2}\left(h\left(y_{0}\right)(a)\right)$. Thus for every $b \in F, \psi_{1}(b)=\psi_{2}(b)$, since $h\left(y_{0}\right)$ is onto. Therefore, $\psi_{1}=\psi_{2}$. For the surjectivity of $\lambda$, let $\varphi \in M(E)$ and note that $\varphi \circ h\left(y_{0}\right)^{-1} \in M(F)$. Then

$$
\lambda\left(\varphi \circ h\left(y_{0}\right)^{-1}\right)=\varphi \circ h\left(y_{0}\right)^{-1} \circ h\left(y_{0}\right)=\varphi .
$$

Hence $\lambda$ is onto and moreover, since $M(E)$ and $M(F)$ are compact Hausdorff spaces, $\lambda^{-1}$ is continuous. Therefore, $\lambda$ is a homeomorphism and hence $M(E)$ is homeomorphic to $M(F)$.

Acknowledgement. We are grateful to the referee who carefully read the paper and made valuable comments and suggestions, which greatly improved the article.

\section{REFERENCES}

1. Y.A. Abramovich and A.K. Kitover, Inverses of disjointness preserving operators, Mem. Amer. Math. Soc., 143 (2000), no. 679, 1-162.

2. Y.A. Abramovich, A. I. Veksler and A.V. Koldunov, Operators preserving disjointness, Dokl. Akad. Nauk USSR, 248 (1979), 1033-1036.

3. J. Araujo and K. Jarosz, Separating maps on spaces of continuous functions, Contemp. Math. 232 (1999), 33-37.

4. W. Arendt, Spectral properties of Lamperti operators, Indiana Univ. Math. J. 32 (1983), 199-215. 
5. E. Beckenstein and L. Narici, A nonarchimedean Stone-Banach theorem, Proc. Amer. Math. Soc. 100 (1987), 242-246.

6. E. Beckenstein and L. Narici, Automatic continuity of certain linear isomorphisms, Acad. Roy. Belg. Bull. Cl. Sci. 73 (1987), no. 5, 191-200.

7. E. Beckenstein and L. Narici, Automatic continuity of linear maps of spaces of continuous functions, Manuscr. Math. 62 (1988), 257-275.

8. H.X. Cao, J. H. Zhang and Z.B. Xu, Characterizations and extensions of Lipschitz- $\alpha$ operators, Acta Math. Sin. (Engl. Ser.) 22 (2006), no. 3, 671-678.

9. H.G. Dales, Banach Algebras and Automatic Continuity, LMS Monographs 24, Clarendon Press, Oxford, 2000.

10. K. Esmaeili and H. Mahyar, Weighted composition operators between vector-valued Lipschitz function spaces, Banach J. Math. Anal. 7 (2013), no. 1, 59-72.

11. J.J. Font, Automatic continuity of certain isomorphisms between regular Banach function algebras, Glasg. Math. J. 39 (1997), 333-343.

12. J.J. Font and S. Hernandez, On separating maps between locally compact spaces, Arch. Math. (Basel) 63 (1994), 158-165.

13. H-L. Gau, J-S. Jeang and N-C. Wong, Biseparating linear maps between continuous vectorvalued function spaces, J. Aust. Math. Soc. 74 (2003), 101-109.

14. M. Hosseini and F. Sady, Banach function algebras and certain polynomially normpreserving maps, Banach J. Math. Anal. 6 (2012), no. 2, 1-18.

15. K. Jarosz, Automatic continuity of separating linear isomorphisms, Canad. Math. Bull. 33 (1990), 139-144.

16. A. Jimenez-Vargas, Disjointness preserving operators between little Lipschitz algebras, J. Math. Anal. Appl. 337 (2008), 984-993.

17. A. Jimenez-Vargas and Ya-Shu Wang, Linear biseparating maps between vector-valued little Lipschitz function spaces, Acta Math. Sin. (Engl. Ser.) 26 (2010), no. 6, 1005-1018.

18. E. Kaniuth, A Course in Commutative Banach Algebras, Springer, Graduate Texts in Mathematics 246, 2009.

19. J.S. Manhas, Weighted composition operators and dynamical systems on weighted spaces of holomorphic functions on Banach spaces, Ann. Funct. Anal. 4 (2013), no. 2, 58-71.

20. A. Nikou and A.G. O'Farrell, Banach algebras of vector-valued functions, Glasgow Math. J., to appear, arXiv:1305.2751.

21. D.R. Sherbert, The Structure of ideals and point derivations in Banach algebras of Lipschitz functions, Trans. Amer. Math. Soc. 111 (1964), 240-272.

22. B.Z. Vulkh, On linear multiplicative operations, Dokl. Akad. Nauk USSR 41 (1943), 148151.

23. B.Z. Vulkh, Multiplication in linear semi-ordered spaces and its application to the theory of operations, Mat. Sbornik 22 (1948), 267-317.

Department of Mathematics, kharazmi University (Tarbiat Moallem UniverSity), 50 Taleghani Avenue, 15618 Tehran, Iran.

E-mail address: honary@khu.ac.ir

E-mail address: std_nikou@khu.ac.ir

E-mail address: a_sanatpour@khu.ac.ir 